BMJ Open

Diabetes

Research

\& Care

\section{Vasomodulation of peripheral blood flow by focused ultrasound potentiates improvement of diabetic neuropathy}

To cite: Tan J-S, Lin C-C, Chen G-S. Vasomodulation of peripheral blood flow by focused ultrasound potentiates improvement of diabetic neuropathy. BMJ Open Diab Res Care 2020;8:e001004. doi:10.1136/ bmjdrc-2019-001004

- Additional material is published online only. To view please visit the journal online (http://dx.doi.org/10.1136/ bmjdrc-2019-001004).

Received 22 October 2019 Revised 31 January 2020 Accepted 22 February 2020

Check for updates

(c) Author(s) (or their employer(s)) 2020. Re-use permitted under CC BY-NC. No commercial re-use. See rights and permissions. Published by BMJ.

${ }^{1}$ Department of Biomedical Engineering and Nanomedicine, National Health Research Institutes, Zhunan, Taiwan ${ }^{2}$ Department of Neurology, National Cheng Kung University Hospital, Tainan, Taiwan

Correspondence to Dr Gin-Shin Chen; gschen@nhri.edu.tw

\section{ABSTRACT}

Objective Effective treatment methods for diabetic peripheral neuropathy are still lacking. Here, a focused ultrasound (FUS) technique was developed to improve blood flow in diabetic peripheral vessels and potentially treat diabetic peripheral neuropathy.

Research design and methods Male adult SpragueDawley rats at 4 weeks poststreptozotocin injections were adopted as models for diabetic neuropathic rats. For single FUS treatment, blood perfusion in the skin of the pad of the middle toe was measured before, during, and after the medial and lateral plantar arteries were treated by FUS. For multiple FUS treatments, blood perfusion measurements, von Frey and hot plate testing and nerve conduction velocity measurements were performed before ultrasonic treatment on the first day of each week, and the microvascular and neural fiber densities in the pad of the toe were measured on the first day of the last week. Results The blood perfusion rate significantly increased for 7-10 min in the control and neuropathic rats after a single ultrasound exposure. Multiple ultrasound treatments compared with no treatments significantly increased blood perfusion at the second week and further enhanced perfusion at the third week in the neuropathic rats. Additionally, the paw withdrawal force and latency significantly increased from $34.33 \pm 4.55 \mathrm{~g}$ and $3.96 \pm 0.25$ $\mathrm{s}$ at the first week to $39.10 \pm 5.02 \mathrm{~g}$ and $4.77 \pm 0.71 \mathrm{~s}$ at the second week and to $41.13 \pm 2.57 \mathrm{~g}$ and $5.24 \pm 0.86 \mathrm{~s}$ at the third week, respectively. The low nerve conduction velocity in the diabetic rats also improved after the ultrasound treatments. Additionally, ultrasound treatments halted the decrease in microvessel and neural fiber densities in the skin of the diabetic toes. Histologic analysis indicated no damage to the treated arteries or neighboring tissue. Conclusions FUS treatment can increase upstream arterial blood flow in diabetic feet, ameliorate the decrease in downstream microvessel perfusion and halt neuropathic progression.

\section{INTRODUCTION}

The global prevalence and incidence of diabetes mellitus were 437-522 million and 21-25 million, respectively, in 2017. ${ }^{1}$ Over $50 \%$ of patients with diabetes can develop diabetic peripheral neuropathy, ${ }^{2}{ }^{3}$ and the most common presentation is distal symmetric polyneuropathy (DSP). ${ }^{4}$ Patients with diabetic DSP often suffer from pain, numbness or

\section{Significance of this study}

What is already known about this subject?

- A vicious cycle between hyperglycemia and microvascular dysfunction can worsen diabetic peripheral neuropathy, particularly distal symmetric polyneuropathy.

- Effective treatment options for diabetic peripheral neuropathy are still lacking.

What are the new findings?

- Focused ultrasound (FUS) treatment on plantar vessels significantly improves diabetic microcirculation.

- FUS therapy concurrently halts diabetes-evoked mechanical allodynic and heat hyperalgesic progression.

- FUS therapy also halts the decrease in the capillary and neural fiber densities in the skin of the pad of diabetic toes.

How might these results change the focus of research or clinical practice?

- Non-invasive FUS treatment potentiates the alleviation of neuropathic pain and/or the prevention of diabetic foot ulcers.

weakness, and some patients even suffer from anxiety, sleep disturbances or foot ulceration and subsequent amputation, leading to a significantly negative impact on their quality of life and an economic burden. ${ }^{5-7}$ Good glycemic control only halts the development of neuropathy in patients with type 1 diabetes and does not affect that of neuropathy in patients with type 2 diabetes. ${ }^{8-10}$ Pain relief can treat symptoms but does not cure DSP. $^{11}$ Medications based on pathogenetic therapies, such as $\alpha$-lipoic acid, benfotiamine, aldose-reductase inhibitors, protein kinase $\mathrm{C}$ inhibitors, nerve growth factors and Actovegin, have been evaluated in clinical trials. ${ }^{12}$ However, none of the medications have been approved by the US Food and Drug Administration or European Medicines Agency.

Focused ultrasound (FUS) is a non-invasive, localized, non-ionizing radiation treatment that has been clinically approved to treat 
brain disorders. ${ }^{13} 14$ Our previous preclinical studies on diabetic peripheral neuropathy demonstrated that FUS acutely and temporarily blocks the conduction of in vitro sural nerves ${ }^{15}$ and compound muscle action potentials of in vivo sciatic nerves in rats with diabetic neuropathy in pain relief applications. ${ }^{16}$ To develop a novel and effective method of treating diabetic DSP, the present study investigated the effects of FUS on nerve-accompanying blood vessels and examined the feasibility of curing DSP in neuropathic rats. We hypothesized that: (1) the perfusion rate of downstream blood vessels can be enhanced when FUS acts on upstream arteries, (2) the improvement of perfusion is applicable to both normal and neuropathic nerves and (3) the improvement in perfusion can partially restore sensory normality and halt DSP progression. Blood perfusion in the pad of the middle toe was analyzed before and after the plantar vessels were exposed to ultrasound. Additionally, the chronic effects of FUS on the blood perfusion, paw withdrawal force, withdrawal latency, nerve conduction velocity, skin microvascular density and neural fiber density of sham and DSP rats were examined.

\section{METHODS}

\section{Animal subjects}

All animal procedures were approved by the Institutional Animal Care and Use Committee of the National Health Research Institutes in Taiwan (AAALAC accredited). Diabetes was induced in male adult Sprague-Dawley rats weighing $300-400 \mathrm{~g}$ by a single intraperitoneal injection of $50 \mathrm{mg} / \mathrm{kg}$ streptozotocin (STZ; Sigma, St. Louis, Missouri, USA). The onset of diabetes was defined as three consecutive measurements of a fasting blood glucose level of $>150 \mathrm{mg} / \mathrm{dL}$ after the STZ injections. The rats exhibited peripheral neuropathy at the fourth week after the STZ injections (online supplementary figure 2). ${ }^{17}$

\section{FUS transducer}

A tailor-made FUS transducer (figure 1A) was used on the plantar arteries of the rats. The transducer was a $2 \mathrm{MHz}$ spherical bowl with a radius of curvature of $16 \mathrm{~mm}$ and an aperture of $20 \mathrm{~mm}$. The front end of the transducer was a hollow cone coupler, and the cone was filled with degassed water and sealed with a $0.03 \mathrm{~mm}$ thick Mylar film. The ellipsoidal focus of the transducer was located at a site $5 \mathrm{~mm}$ beneath the Mylar film, and the focal width and depth were $0.6 \mathrm{~mm}$ and $2.4 \mathrm{~mm}$, respectively. The transducer was driven by a function generator (33 521A, Agilent, Santa Clara, California, USA) and a power amplifier $(1040$ L, Electronics and Innovation, Rochester, New York, USA) in all experiments.

\section{Measurements of blood perfusion}

The rats were anesthetized by the inhalation of isoflurane $(3 \%-4 \%$ for induction and $1.25 \%-1.75 \%$ for maintenance, Panion \& BF Biotech Inc, Taoyuan, Taiwan) in an oxygen mixture. The anesthetized rats were placed on a custom-made table in the ventral recumbent position,
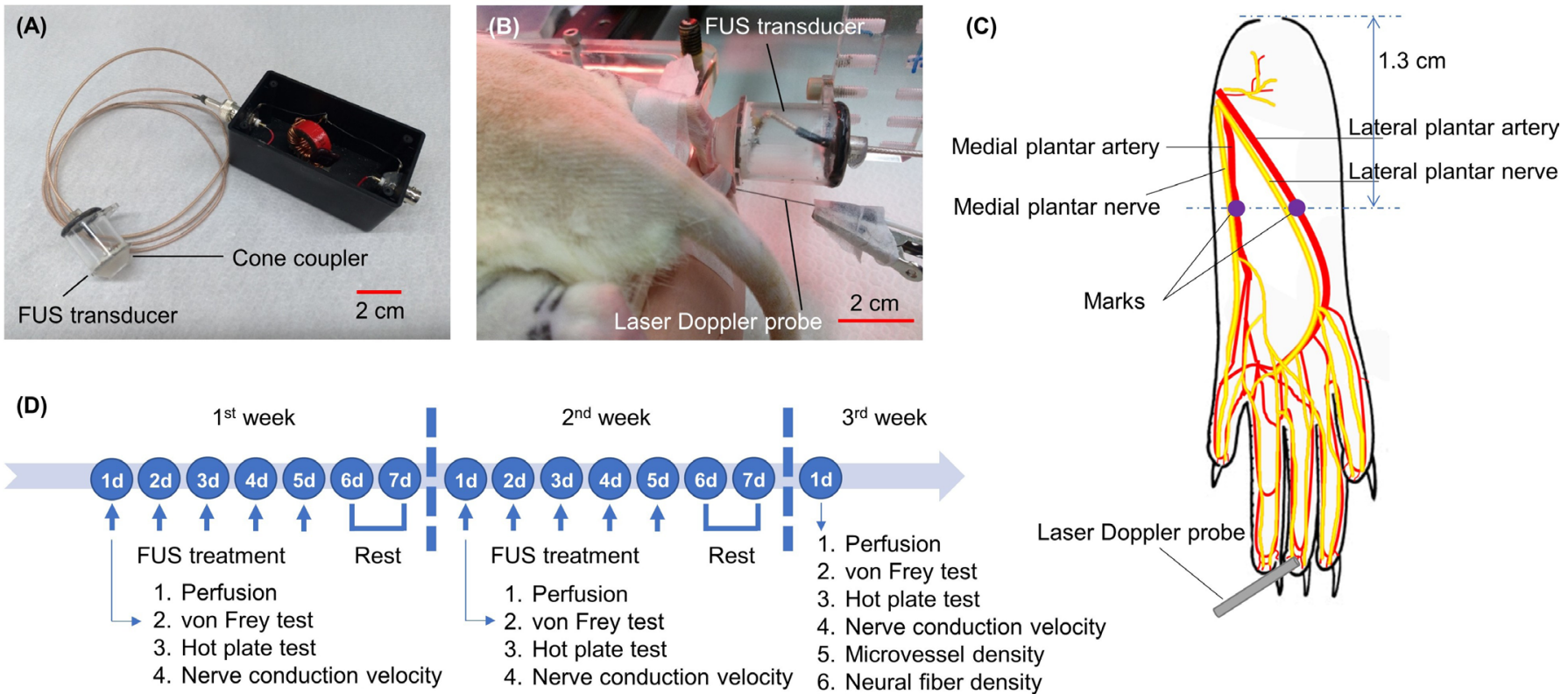

Figure 1 The experimental setup and design. (A) A custom-made focused ultrasound transducer consisting of a spherically piezoelectrical ceramic, an acrylic cone coupler, housing, electrical connections and circuits was made. (B) The plantar artery of the anesthetized rat was treated by focused ultrasound, and the blood perfusion in the skin of the pad of the middle toe was measured by the laser Doppler instrument. (C) The drawing depicts the locations of the locally treated arteries. The mark on the skin of the sole was the contact point of the ultrasound transducer. (D) For multiple ultrasound treatments, ultrasound exposure was carried out once a day for the first 5 days per week for 2 weeks. Blood perfusion measurements from the end of the middle toe and von Frey testing were performed before ultrasonic treatment on the first day of each week. The microvascular density of the skin on the end of the toe was measured on the first day of the third week. FUS, focused ultrasound. 
and both feet were suspended off of the table. The soles of the feet were facing outward, and the five toes of each foot were fixed with tape. The probe (diameter of 0.5 $\mathrm{mm}, \mathrm{TSD} 145$ ) of the laser Doppler flowmeter (LDF100c, Biopac Systems, Inc, Goleta, California, USA) was placed so that it gently touched the skin of the pad of the middle toe and blood perfusion could be measured (figure 1B). The data were acquired by the system (Biopac Systems, Inc) at a sampling rate of $200 \mathrm{~Hz}$. Laser Doppler signals from the vessel were recorded in blood perfusion units (BPUs).

\section{Single FUS treatment}

The rats $(n=12)$ were randomly divided into two groups: the Control I rats, which received no STZ injections $(\mathrm{n}=6)$, and DSP I rats, which received an STZ injection 4 weeks prior $(n=6)$. The light penetrated the rat foot from the instep to the sole so that the medial plantar and lateral plantar arteries were visualized, and two marks that were projected to the two arteries were made on the skin of the sole to position the FUS transducer. The distance between the mark and the ankle was approximately 1.3 $\mathrm{cm}$ in all experiments (figure 1C). Ultrasonic gel spread on the skin was used as the acoustic medium, and the medial and lateral plantar arteries were treated once with FUS. Before each plantar artery was treated, the blood perfusion in the end of the middle toe was measured for $60 \mathrm{~s}$ as the baseline. For both groups, the left and right feet of every rat were treated sequentially using the same protocol. Ultrasound with a spatial-average temporalaverage intensity of $1126 \mathrm{~W} / \mathrm{cm}^{2}$ and sonication of $15 \mathrm{~s}$ was used in each treatment. FUS with the above parameters can cause a temperature increase by $2^{\circ} \mathrm{C}$ in the focus area in the muscle-mimicking phantom (online supplementary figure 1 ).

\section{Multiple FUS treatments}

The remaining rats $(n=12)$ were randomly divided into two groups: control II rats, which received no STZ injections $(n=6)$, and DSP II rats, which received an STZ injection 4 weeks previously $(n=6)$. The same anesthesia, positioning, equipment and ultrasonic parameters as those described for the single FUS treatment were used. For the DSP II rats, FUS heated the medial plantar artery of the right foot once and then the lateral plantar artery once with an interval of $5 \mathrm{~min}$ (DSP II+FUS group), and the left foot of the same rat was regarded as the DSP II sham group. The FUS treatment was carried out once a day for the first 5 days per week for 2 weeks. Blood perfusion measurements in the pad of the middle toe, pain assays and nerve conduction velocity measurements were performed before ultrasonic treatment on the first day of each week, and the microvascular density and the neural fiber density of the toe end skin were measured on the first day of the third week (figure 1D). For the control II rats, the experimental procedure was the same as that for the DSP II sham group.

\section{Neuropathic pain assays}

Mechanical allodynia and heat hyperalgesia are usually used as neuropathic indicators and assessed with the von Frey test and hot plate test, respectively. ${ }^{17}$ Accordingly, the DSP of the rats that were administered multiple FUS treatments was evaluated using an electronic von Frey device (IITC 2390, IITC Life Science, Woodland Hills, USA) and a hot plate analgesia meter (IITC Life Science) in the present study. The plantar surface of the rat hind paw was stimulated with a rigid tip that was $0.8 \mathrm{~mm}$ in diameter. The force was increased gradually until the rat withdrew its paw, and the von Frey anesthesiometer was used to record the withdrawal force. To acclimatize them to the environment, the rats were placed on the hot plate at a temperature of $24 \pm 0.5^{\circ} \mathrm{C}$ for $10 \mathrm{~min}$ before the experiment. Each rat was then removed and placed back on the hot plate when the plate was heated to and maintained at $55 \pm 0.5^{\circ} \mathrm{C}$. The rats expressed distinct behaviors, such as licking the hind paw or abnormally flicking the hind paw, when they felt pain. The withdrawal latency was defined as the time to withdrawal. Five measurements of the withdrawal force and the withdrawal latency were repeated for each rat.

\section{Nerve conduction velocity}

The compound nerve action potential of the plantar nerve was recorded with a pair of stainless-steel acupuncture needle electrodes. The anodic recording electrode was inserted at the ankle behind the medial malleolus, and the cathodic electrode was placed $1 \mathrm{~cm}$ proximal to the anodic electrode. ${ }^{18}$ Two ring silver wires (UL1423 28 AWG B28-1000, AA electronic Test, USA) were placed on the middle three digits as stimulating electrodes to deliver an electrical stimulus with a pulse width of 0.1 ms and a supramaximal intensity. Nerve stimulation and recording were performed using the Biopac MP36 instrument (Biopac Systems, Inc). The nerve conduction velocity was calculated as the distance divided by the latency of the negative peak of the compound action potentials, where the distance between the cathodic stimulating and anodic recording electrodes was measured with a Vernier caliper.

\section{Intraepidermal microvascular density and nerve fiber density}

The rats were sacrificed by $\mathrm{CO}_{2}$ inhalation under anesthesia at the end of the single or multiple FUS treatments. Epidermis-to-dermis tissues from the pads of the second, middle and fourth toes were harvested, fixed in $10 \%$ formalin and embedded in paraffin for immunohistochemical staining. The primary antibody of monoclonal anti-CD34 antibody (ab81289, Abcam, Cambridge, Massachusetts, USA) prepared at a 1/200 dilution for 1 hour at room temperature and the secondary antibody of Discovery OmniMap anti-Rb HRP (760-4311, Ventana Medical Systems, Tucson, Arizona, USA) with an ultraView universal DAB detection kit (760-500, Ventana Medical Systems) were used to stain the vascular endothelial cells brown. The primary and secondary antibodies 
used to stain the neural fibers brown were the monoclonal anti-PGP9.5 antibody (ab8189, Abcam) prepared at a $1 / 1000$ dilution for 1 hour at room temperature and Discovery OmniMap anti-Rb HRP with an ultraView universal DAB detection kit, respectively. ${ }^{19}$ The endothelial cells and fibers were stained by the Pathology Core Laboratory at the National Health Research Institutes. The tissue sections were scanned at magnifications of $100 \times$ to identify the most vascular regions in the dermis. Within these regions, 10 fields $\left(0.0336 \mathrm{~mm}^{2}\right.$ per field) were inspected, and immunolabeled microvessels were counted in a $400 \times$ field..$^{20}$ Image J software $(1.50 \mathrm{i}$, National Institutes of Health, USA) was used to automatically select and count the brown microvessels by setting the color threshold hue to 60 and analyzing particles with sizes $>30 \mu \mathrm{m}^{2}{ }^{21}$ For the nerve fiber density, microphotographs of the stained sections were taken on a microscope at $40 \times$ magnification, and the length of the epidermis was assessed with ImageJ software. The intraepidermal nerve fiber profiles were counted by three independent researchers blinded to the study groups.

\section{Histology}

Histological analysis was performed to examine the safety of the FUS dose used in the present study. The rats were sacrificed by $\mathrm{CO}_{2}$ inhalation under anesthesia after 2 weeks of FUS exposure. The tissue treated by FUS was harvested, fixed in $10 \%$ formalin and then stained with H\&E by a technician blinded to the treatments of the rats.

\section{Statistical analyses}

All experimental data are expressed as the mean \pm SD. When data demonstrated a normal distribution, oneway analysis of variance followed by Duncan's test for post hoc comparisons was used for analysis. Otherwise, non-parametric analysis using the Kruskal-Wallis test and Mann-Whitney $\mathrm{U}$ test for post hoc paired comparisons was performed. The levels of statistical significance was set to be ${ }^{*} \mathrm{p}<0.05$ and ${ }^{* *} \mathrm{p}<0.01$.

\section{RESULTS}

\section{Changes in microcirculation}

The blood perfusion in the ends of the right and left middle toes in the DSP I rats $(255 \pm 48 \mathrm{BPU})$ was significantly lower than that in the control I rats $(479 \pm 26 \mathrm{BPU})$ before single FUS treatment (online supplementary figure 3). Online supplementary figure 3 illustrates one example of a skin blood perfusion profile for a DSP I rat subjected to single ultrasound treatment. The baseline blood perfusion level and the duration of increased perfusion (T) were $203 \pm 38$ BPU and $144 \mathrm{~s}$, respectively. One FUS sonication to the lateral plantar arteries in the control I rats or DSP I rats resulted in a significant increase in blood perfusion in the ends of the middle toes (figure 2A), where the blood perfusion was increased from $523 \pm 51$ to $1070 \pm 346 \mathrm{BPU}$ for the control I rats and from $255 \pm 53$ to $890 \pm 312$ BPU for the DSP I rats. Similar
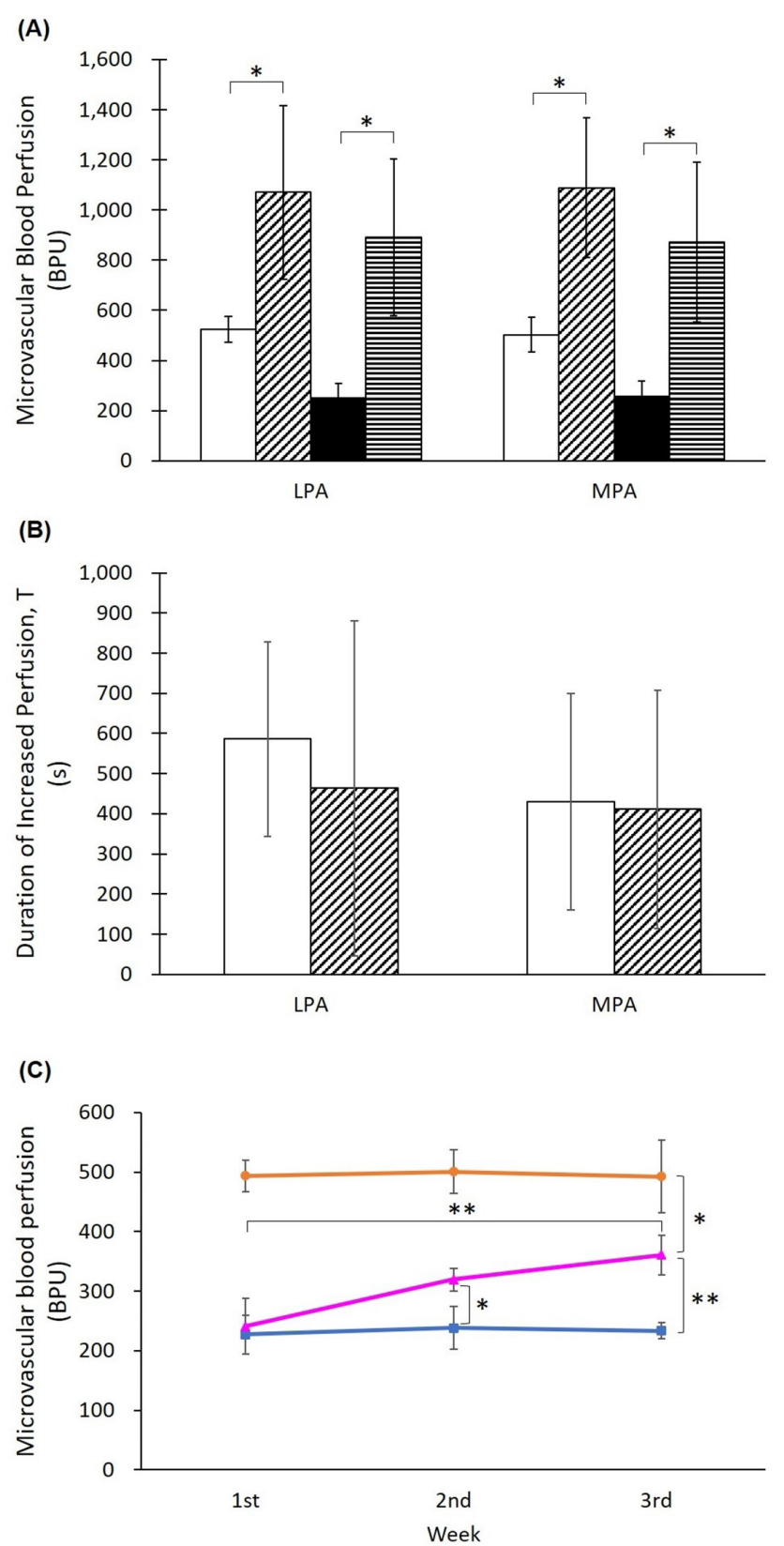

Figure 2 The microvascular blood perfusion response in the skin of the toe to a single ultrasound exposure on plantar arteries. (A) Skin blood perfusion increased significantly after the lateral plantar artery (LPA) or medial plantar artery (MPA) was treated by ultrasound in the control and DSP rats. Blank bar=control I before FUS; slash bar=control I after FUS; black bar=DSP I before FUS; hyphen bar=DSP I after FUS. ${ }^{*}: P<0.05$. (B) The duration of increased blood perfusion was 7-10 min in both the control and DSP rats. The difference in the duration was not statistically significant among the four conditions. Blank bar=control I; slash bar=DSP I. (C) Effect of multiple ultrasound treatments on skin blood perfusion in plantar arteries. Microvascular blood perfusion in the skin of the toe increased significantly after 1 week of multiple ultrasound sonication to the plantar arteries and further increased after another week of treatment in the DSP rats. Orange circle=control II group; blue square=DSP II sham group; pink triangle=DSP $\|+F U S$ group. ${ }^{*} \mathrm{P}<0.05$; ${ }^{\star *} \mathrm{p}<0.01$. BPU, blood perfusion uni; DSP, distal symmetric polyneuropathy; FUS, focused ultrasound. 
(A)

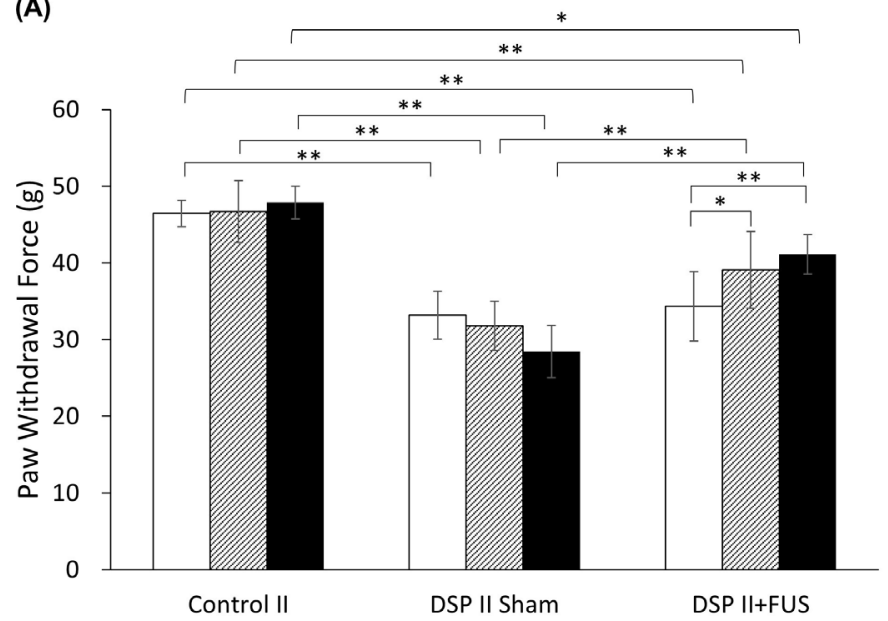

(B)

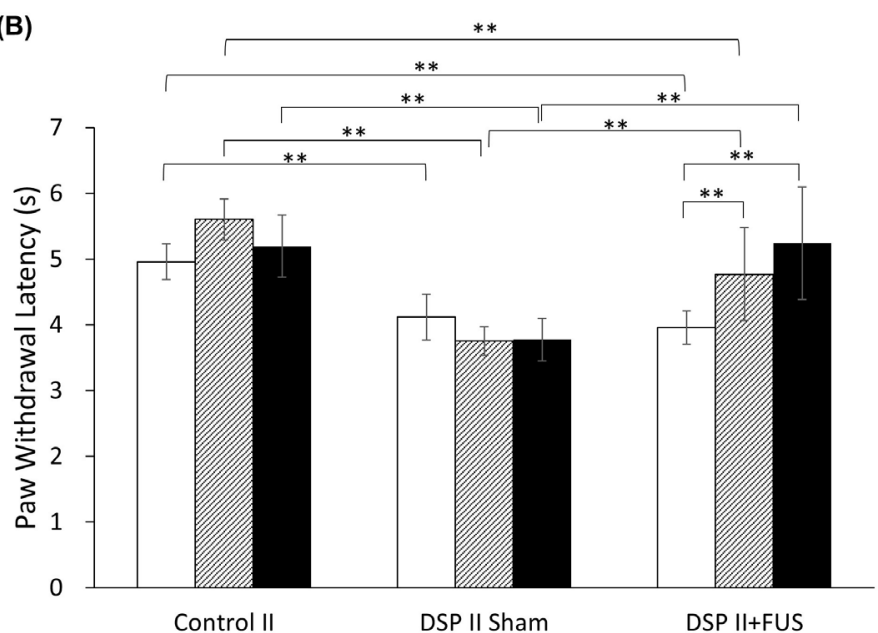

Figure 3 Effect of multiple ultrasound treatments on plantar arteries on mechanical allodynia (A) and heat hyperalgesia (B). For the control rats, the paw withdrawal force and latency did not vary significantly over 2 weeks. The withdrawal force and latency of the DSP rats were remarkably low compared with those of the control rats. One-week multiple ultrasound treatments in the DSP rats (DSP II+FUS group) led to a significant increase in the withdrawal force and latency compared with the untreated DSP rats (DSP II sham group). Two-week ultrasound treatments further improved the withdrawal response and latency. Blank bar=first week; slash bar=second week; black bar=third week. ${ }^{\star} P<0.05$; ${ }^{\star \star} p<0.01$. DSP, distal symmetric polyneuropathy; FUS, focused ultrasound.

phenomena occurred for the medial plantar arteries. The perfusion increased significantly from $502 \pm 69$ BPU and $259 \pm 58 \mathrm{BPU}$ at baseline to $1088 \pm 278 \mathrm{BPU}$ and $872 \pm 317$ BPU for the control I rats and DSP I rats, respectively, after single FUS treatment. In addition, increased skin blood perfusion lasted $586 \pm 243 \mathrm{~s}$ and $429 \pm 270 \mathrm{~s}$ in the lateral and medial plantar arteries, respectively, of the control I rats and $464 \pm 418 \mathrm{~s}$ and $411 \pm 296 \mathrm{~s}$ in the lateral and medial plantar arteries, respectively, of the DSP I rats (figure 2B). Differences in the duration between the above four cases were not significant.

For multiple FUS treatments, blood perfusion in the ends of the middle toes in the DSP II sham group and DSP II+FUS group were $242 \pm 36$ BPU and $266 \pm 45 \mathrm{BPU}$, respectively, at the first week before FUS treatment (figure 2C). After multiple treatments, the perfusion of the DSP II+FUS group increased significantly to $323 \pm 21$ BPU at the second week and additionally increased to $360 \pm 47$ BPU at the third week compared with those of the DSP II sham group. For the DSP II sham group, the perfusion decreased with time but did not change significantly in the 2 weeks. For the DSP II+FUS group, the perfusion of the third week was significantly larger than that of the first week but still lower than that of the control II group $(493 \pm 26 \mathrm{BPU}$ at the first week, $500 \pm 37 \mathrm{BPU}$ at the second week and $492 \pm 61 \mathrm{BPU}$ at the third week).

\section{Change in mechanical allodynia and heat hyperalgesia}

The paw withdrawal force of the DSP II sham group $(33.20 \pm 3.13 \mathrm{~g}$ at the first week, $31.79 \pm 3.18 \mathrm{~g}$ at the second week and $28.45 \pm 3.39 \mathrm{~g}$ at the third week) was significantly lower than that of the control II group $(46.59 \pm 1.71$ $\mathrm{g}, 46.98 \pm 4.04 \mathrm{~g}$ and $48.06 \pm 2.16 \mathrm{~g}$ ) (figure $3 \mathrm{~A}$ ). For the DSP II+FUS group, the paw withdrawal force significantly increased from $34.33 \pm 4.55 \mathrm{~g}$ at the first week to $39.10 \pm 5.02$ $\mathrm{g}$ at the second week and to $41.13 \pm 2.57 \mathrm{~g}$ at the third week. Furthermore, the third week withdrawal force of the DSP II+FUS group was significantly higher than that of the DSP II sham group and was similar to that of the control II group. The paw withdrawal latency and the paw withdrawal force had similar trends in the control II, DSP II sham and DSP II+FUS groups (figure 3B). The paw withdrawal latency of the DSP II sham group $(4.12 \pm 0.35 \mathrm{~s}$ at the first week, $3.75 \pm 0.22 \mathrm{~s}$ at the second week and $3.77 \pm 0.33 \mathrm{~s}$ at the third week) was significantly lower than that of the control II group $(4.96 \pm 0.27 \mathrm{~s}$, $5.60 \pm 0.31 \mathrm{~s}$ and $5.19 \pm 0.47 \mathrm{~s})$. The paw withdrawal latency

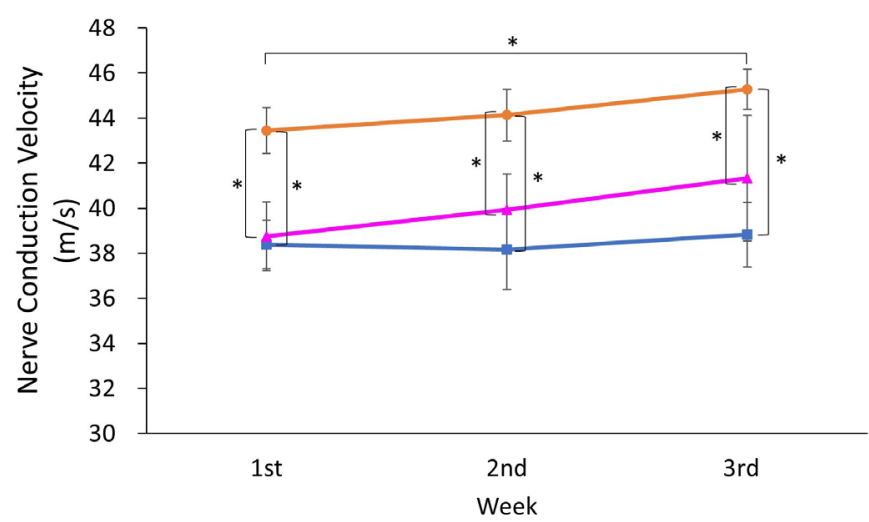

Figure 4 Effect of multiple ultrasound treatments on plantar arteries on plantar nerve conduction velocity. The nerve conduction velocity of the DSP rats was significantly lower than that of the control rats. The velocity of the DSP rats (DSP II+FUS group) increased after multiple ultrasound treatments, whereas the untreated DSP rats had a nearly constant velocity over 2 weeks. Orange circle=control II group; blue square=DSP II sham group; pink triangle=DSP II+FUS group. ${ }^{*} \mathrm{P}<0.05$. DSP, distal symmetric polyneuropathy; FUS, focused ultrasound. 
(A)

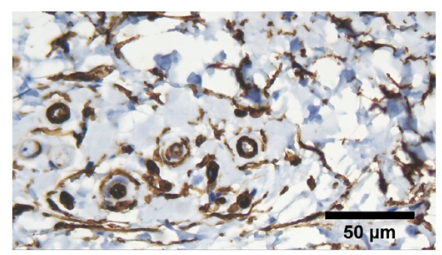

(C)

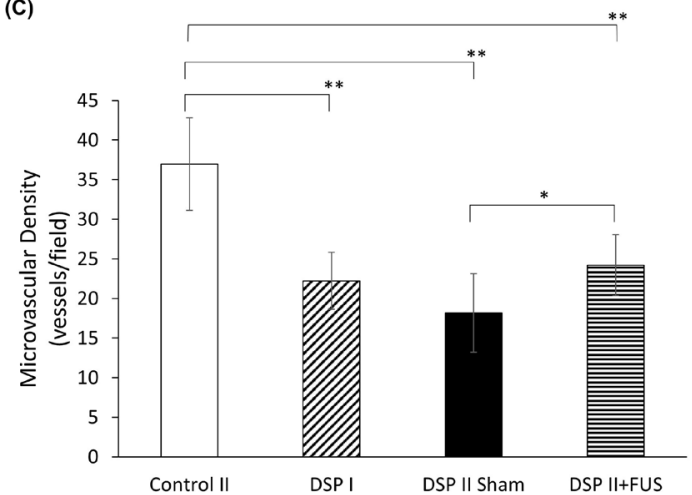

(B)

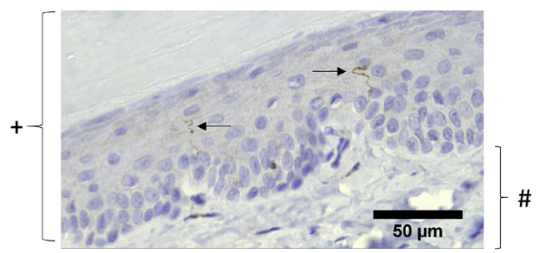

(D)

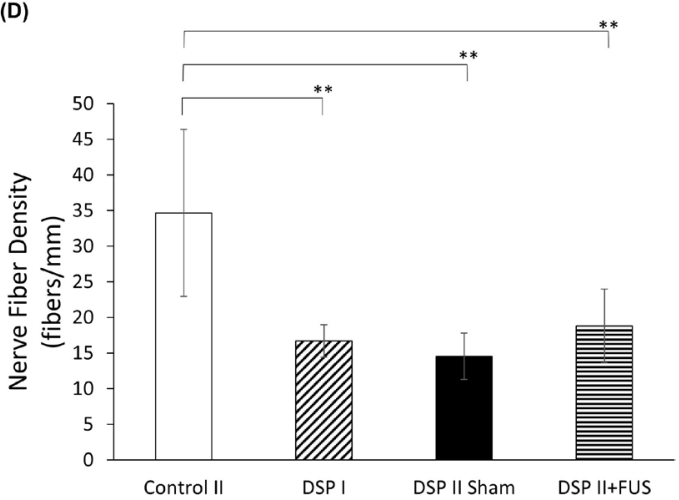

Figure 5 Analysis of microvessel density and nerve fiber density in three-toe skin. The two representative pictures of immunostained skin illustrate brown vascular endothelial cells $(\mathrm{A})$ and neural fibers $(\mathrm{B})$. The brown area $>30 \mu \mathrm{m}^{2}$ denotes a microvessel. Arrows: neural fiber; +: epidermis; \#: dermis. (C) The skin microvascular density of all the DSP rats was significantly lower than that of the control rats. Among the DSP rats, the rats at 6 weeks after the STZ injection (DSP II sham group) had a low density compared with the rats at 4 weeks after the STZ injection (DSP I group), but the 6-week rat subjected to a 2-week ultrasound treatment (DSP II+FUS group) had a similar density to the 4-week rats. (D) The nerve fiber density of the DSP I, DSP II sham and DSP II+FUS groups was significantly lower than that of the control II group. Among the DSP I, DSP II sham and DSP II+FUS groups, the DSP II+FUS group had the highest density. ${ }^{*} P<0.05 ;{ }^{* *} \mathrm{p}<0.01$. DSP, distal symmetric polyneuropathy; FUS, focused ultrasound; STZ, streptozotocin.

of the DSP II+FUS significantly increased from $3.96 \pm 0.25$ $\mathrm{s}$ at the first week to $4.77 \pm 0.71 \mathrm{~s}$ at the second week and to $5.24 \pm 0.86 \mathrm{~s}$ at the third week. Furthermore, the third week withdrawal latency of the DSP II+FUS group was significantly higher than that of the DSP II sham group and was almost the same as that of the control II group.

\section{Change in nerve conduction velocity}

The nerve conduction velocity of the DSP II sham group $(38.39 \pm 1.08 \mathrm{~m} / \mathrm{s}$ at the first week, $38.16 \pm 1.77 \mathrm{~m} / \mathrm{s}$ at the second week and $38.82 \pm 1.43 \mathrm{~m} / \mathrm{s}$ at the third week) and DSP II+FUS group $(38.75 \pm 1.53 \mathrm{~m} / \mathrm{s}, 39.92 \pm 1.59 \mathrm{~m} / \mathrm{s}$ and $41.33 \pm 2.79 \mathrm{~m} / \mathrm{s})$ was significantly lower than that of the control II group $(43.44 \pm 1.01 \mathrm{~m} / \mathrm{s}, 44.13 \pm 1.15 \mathrm{~m} / \mathrm{s}$ and $45.28 \pm 0.89 \mathrm{~m} / \mathrm{s}$ ) (figure 4 ). The velocity of the DSP II+FUS group gradually increased with time and was higher than that of the DSP II sham group.

\section{Change in skin microvessel density and nerve fiber density}

Immunostaining turned the vascular endothelial cells and neural fibers brown, as shown in figure $5 \mathrm{~A}$ and $\mathrm{B}$. The numbers of microvessels in the skin of the middle three toes were $37 \pm 6,22 \pm 4,18 \pm 5$ and $24 \pm 4$ per field for the control II group (6-week rats without STZ injection), the DSP I group (4-week STZ rats treated by single FUS), the DSP II sham group (6-week STZ rats) and the DSP II+FUS group (4-week STZ rats treated by the following 2-week FUS), respectively. Compared with the control II group, the DSP I, DSP II sham and DSP II+FUS groups had significantly lower microvascular density (figure 5C). In addition, the density of the DSP II+FUS group was significantly higher than that of the DSP II sham group and similar to that of DSP I rats. For the nerve fiber density, the epidermal length of the three-toe skin sample was $2.8 \pm 0.1 \mathrm{~mm}$, and the number of fibers were $35 \pm 12,17 \pm 2,15 \pm 3$ and $19 \pm 5$ per $\mathrm{mm}$ for the control II group, the DSP I group, the DSP II sham group and the DSP II+FUS group, respectively (figure 5D). The nerve fiber density of the DSP I, DSP II sham and DSP II+FUS groups was significantly lower than that of the control II group. Among the DSP I, DSP II sham and DSP II+FUS groups, the DSP II+FUS group had a highest density.

\section{Staining of the treated tissues}

Histological analysis revealed that the morphology of the skin and lateral plantar tissue in the DSP II+FUS rats (figure 6E and $\mathrm{F}$ ) was similar to that in the DSP II sham rats (figure $6 \mathrm{~A}$ and $\mathrm{B}$ ). The treated lateral plantar artery and the neighboring lateral plantar nerve continued to display an organized structure (figure 6F-1 and F-2) similar to that of the untreated artery and nerve (figure 6B-1 and B-2). Similarly, the histological morphology of the skin, medial plantar tissue, medial plantar artery and neve in the DSP II+FUS rats (figure 6G, H, H-1 and H-2) resembled that in the DSP II sham rats (figure $6 \mathrm{C}, \mathrm{D}, \mathrm{D}-1$ and D-2). Because the distal medial plantar tissue was 

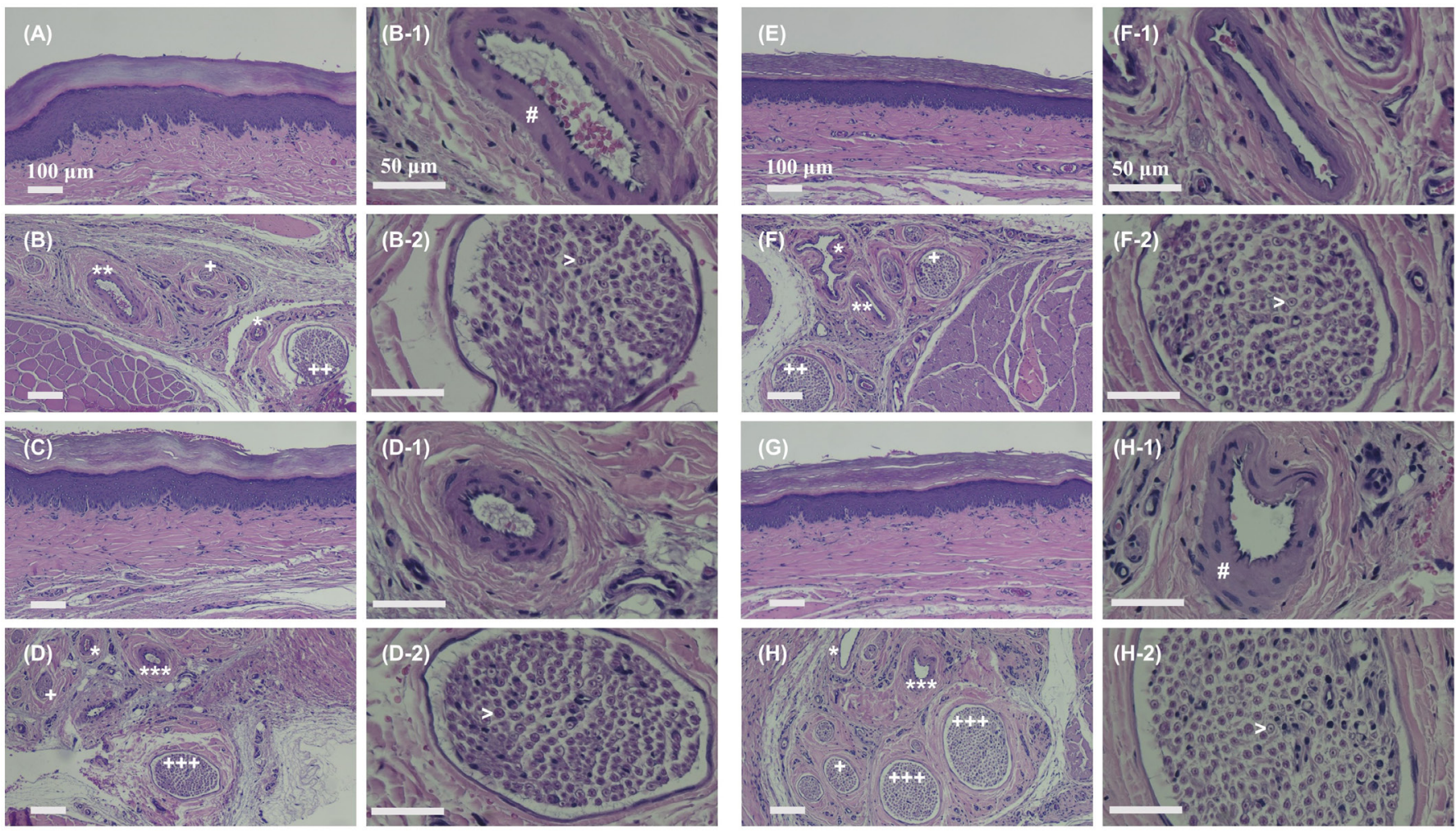

Figure 6 H\&E staining images of rat foot tissue cut in the sagittal plane. Figure parts A-D show the skin above the lateral plantar tissue, lateral plantar tissue, skin above the medial plantar tissue and medial plantar tissue of a DSP rat that did not undergo multiple ultrasound treatments. Figure parts B-1 and B-2 are magnifications of B and D-1 and D-2 are magnifications of $D$, illustrating the lateral plantar artery and nerve ane the medial plantar artery and nerve, respectively. Figure parts $\mathrm{E}-\mathrm{H}$ ) show the skin above the lateral plantar tissue, lateral plantar tissue, skin above the medial plantar tissue and medial plantar tissue of a DSP rat that underwent multiple ultrasound treatments. Figure parts F-1 and F-2 are magnifications of F, and $\mathrm{H}-1$ and $\mathrm{H}-2$ are magnifications of $\mathrm{H}$, illustrating the lateral plantar artery and nerve and the medial plantar artery and nerve, respectively. *: vessel; +: nerve; **: lateral plantar artery; ++: lateral plantar nerve; ${ }^{\star \star *}$ : medial plantar artery; +++: medial plantar nerve; \#: tunica media; >: nerve fiber. DSP, distal symmetric polyneuropathy.

harvested, the medial plantar nerve branches are shown in figure $6 \mathrm{H}$.

\section{DISCUSSION}

Human studies have shown that the disruption of skin microvascular blood flow may be an early manifestation of diabetic autonomic neuropathy, ${ }^{22} 23$ and skin vasomotor function is significantly attenuated in patients with diabetic peripheral or autonomic neuropathy. ${ }^{2425}$ Moreover, nociceptive C fibers secrete substance $\mathrm{P}$ to cause vasodilation, ${ }^{2627}$ and this vasodilation response is decreased in the presence of sensory diabetic neuropathy. ${ }^{28}{ }^{29}$ Accordingly, skin blood perfusion, in addition to mechanical allodynia, heat hyperalgesia and nerve conduction velocity, was adopted as an indicator of DSP assessment.

Acute effectiveness of FUS in improving the skin blood perfusion of the toe was observed in the control and DSP rats when ultrasound was applied to the plantar artery. The increased level of blood perfusion was twofold that at baseline in the control rats and more than threefold that at baseline in the DSP rats after the single FUS treatment. The phenomenon of increased blood perfusion lasted 7-10 min in the control and DSP rats. The lateral and medial plantar arteries connect to four common plantar digital arteries and separate into the proper digital arteries for two adjacent toes. ${ }^{30}{ }^{31}$ Increasing the upstream blood flow probably increases the skin blood perfusion of the middle toe. Therefore, the remarkable results support our assumptions that exposing the upstream arteries to ultrasound can enhance the blood perfusion of downstream or distal small arteries in the toes, and the improvement in perfusion is applicable to both normal and neuropathic nerves. Notably, FUS enables a large increase in perfusion for DSP rats.

Local hyperthermia is one mechanism of increasing blood flow accompanied by the dilation of vessels in normal femoral arteries. ${ }^{32-34}$ These studies have revealed that the temperature increases were $2^{\circ} \mathrm{C}$ and $1{ }^{\circ} \mathrm{C}$ in the vastus lateralis muscle and the femoral vein, respectively. Our study showed that FUS induced an increase in temperature by $2^{\circ} \mathrm{C}$ in the muscle phantom, suggesting local heating in the muscles near the plantar arteries. An estimated increase by $1^{\circ} \mathrm{C}$ in the plantar artery locally exposed to FUS is plausible. In addition, the mechanical effect induced by ultrasound is probably another 
mechanism of vasodilation of the artery. ${ }^{35}$ Ultrasonic pressure waves may stimulate the vascular endothelium to release vasodilator substances such as nitric oxide and prostacyclin, leading to vascular smooth muscle relaxation and vasodilation. ${ }^{36}$ Therefore, the synergy of the thermal and mechanical effects of ultrasound contributes to an increase in the blood flow of plantar arteries.

The chronic effects of FUS significantly ameliorated peripheral micro blood perfusion in DSP rats after the first week of treatment, and further improvement was observed after the 2-week treatment. With respect to mechanical allodynia pain and heat hyperalgesia, a similar phenomenon occurred; that is, after the first week of FUS treatment, the paw withdrawal force and latency of the DSP rats markedly increased, and their paw withdrawal force and latency increased further compared with those of the untreated DSP rats after another week of treatment. Notably, the paw withdrawal force and latency of the DSP II+FUS rats were similar to those of the control II rats by the third week. Vascular impairment and metabolic deficits may be major pathogenetic mechanisms responsible for diabetic neuropathy. ${ }^{37}{ }^{38} \mathrm{In}$ cases of diabetes mellitus, autonomic nerve endings to the epineurial arteriole are likely to be lost, and thus, vasoregulation is impaired in the peripheral nerves. ${ }^{39}$ Impaired autoregulation can cause decreased blood perfusion in transperineurial arterioles and endoneurial capillaries, leading to endoneurial hypoxia and diabetic neuropathy. ${ }^{3740}$ We presume that FUS-enhanced blood flow increases the vascular supply to the endoneurium and nerve fibers through transperineurial arterioles and endoneurial capillaries and further repairs impaired nerve fibers. The improvement in neural function, which breaks the vicious cycle between decreased local blood perfusion and impaired neural regulatory function, may have synergistic beneficial effects.

Most human studies have shown no significant reduction in skin capillary density in patients with early-stage diabetes. ${ }^{41}{ }^{42}$ However, capillary loss may eventually occur in the late stage and manifest as diabetic feet. Interestingly, the skin vessel density significantly decreased in the DSP rat models of the present study in the early stage. Furthermore, we found that 2-week FUS treatments can halt the decrease in the number of microvessels in the DSP rats. Growing evidence shows a vicious cycle of hyperglycemia and microvascular dysfunction. ${ }^{43}$ Hyperglycemia induces microvascular dysfunction, such as a reduced capillary density in muscle, and microvascular dysfunction reduces glucose disposal in muscle and insulin secretion by pancreatic islets, leading to hyperglycemia. Our study demonstrated that FUS treatment of peripheral vessels can enhance blood flow and halt the decrease in capillary density, which may improve cases of hyperglycemia and DSP.

A decrease in the plantar nerve conduction velocity was also ameliorated in the diabetic rats treated with FUS. Diabetes elicits functional and structural changes in the peripheral nerve, decreasing the nerve conduction velocity. Two-week FUS treatments on the peripheral blood vessel may halt the reduction in nodal or paranodal potassium currents in neural axons ${ }^{44}$ and even arrest the reduction in myelin sheath surface area, myelin/ axon ratio and the number of unmyelinated axons or small fibers ${ }^{45}$ due to improved microcirculation of blood, which increases the low conduction velocity.

The thermal dose that caused damage to $50 \%$ of the vessels was $144 \pm 46$ equivalent minutes at $43^{\circ} \mathrm{C}$ for the normal vessels ${ }^{46}$ and the thermal dose of heat-induced cell death was 210-240 min for muscle, fat and skin. ${ }^{47}$ The thermal dose of the plantar vessel induced by FUS (15 s at body temperature $+2^{\circ} \mathrm{C}$ ) is much lower than the above thermal dose of vessel damage. Moreover, the histological analysis shows the undisrupted morphology of the skin, fat, muscle and vessels treated by FUS. Therefore, the FUS parameters used in the study did not cause adverse biological effects.

Rats with short-term diabetes mainly develop pain, whereas those with longer term diabetes typically develop both pain and insensate symptoms. ${ }^{6}$ Hypoalgesia was not evaluated because we believe interventions may be more effective in the early state of neuropathy before irreversible changes appear. Although numbness and hypoesthesia were the major initial symptoms of distal symmetric diabetic polyneuropathy in humans, microangiopathy was the major underlying etiology, similar to that in STZinduced rats. Because improvements in blood perfusion and the number of microvessels and neural fibers were shown in this study, the difference in the initial presentation of neural dysfunction in the animal model may not hinder the contribution of the present study.

We have developed the first FUS technique for modulating diabetic blood vessels and demonstrated that increasing the blood flow of upstream plantar vessels can increase the downstream microcirculation in the skin of toes. Additionally, we showed that allodynic and hyperalgesic pain improve after FUS treatments and that the increases in paw withdrawal force and latency are positively correlated with the increase in the perfusion rate of the skin vessels. FUS treatment also halts the decreases in microvessel density and neural fiber density in the skin of diabetic feet. These findings corroborate the FUS treatment efficacy for diabetic distal polyneuropathy.

Acknowledgements The authors would like to thank the Pathology Core Laboratory of the National Health Research Institutes for the staining work performed in the study.

Contributors J-ST performed the experiments and statistical analyses and drafted the manuscript. C-CL contributed to the discussion and reviewed the manuscript. G-SC designed the study, contributed to the discussion and reviewed/edited the manuscript. G-SC is the guarantor of this work and, as such, had full access to all data in the study and takes responsibility for the integrity and accuracy of the data analysis.

Funding This work was supported by the National Health Research Institutes (BN-108-PP-10) and the Ministry of Science and Technology (MOST 105-2221-E-400-004-MY3 and MOST 108-2221-E-400-001) in Taiwan.

Competing interests None declared.

Patient consent for publication Not required. 
Ethics approval All animal procedures were approved by the Institutional Animal Care and Use Committee of the National Health Research Institutes in Taiwan (AAALAC accredited).

\section{Provenance and peer review Not commissioned; externally peer reviewed.}

Data availability statement All data relevant to the study are included in the article or uploaded as supplementary information.

Open access This is an open access article distributed in accordance with the Creative Commons Attribution Non Commercial (CC BY-NC 4.0) license, which permits others to distribute, remix, adapt, build upon this work non-commercially, and license their derivative works on different terms, provided the original work is properly cited, appropriate credit is given, any changes made indicated, and the use is non-commercial. See: http://creativecommons.org/licenses/by-nc/4.0/.

ORCID iD

Gin-Shin Chen http://orcid.org/0000-0003-3368-7470

\section{REFERENCES}

1 DiseaseGBD, Injuryl, PrevalenceCGlobal, et al. Global, regional, and national incidence, prevalence, and years lived with disability for 354 diseases and injuries for 195 countries and territories, 1990-2017: a systematic analysis for the global burden of disease study 2017. Lancet 2018;392:1789-858.

2 Dyck PJ, Litchy WJ, Lehman KA, et al. Variables influencing neuropathic endpoints: the Rochester diabetic neuropathy study of healthy subjects. Neurology 1995;45:1115-21.

3 Edwards JL, Vincent AM, Cheng HT, et al. Diabetic neuropathy: mechanisms to management. Pharmacol Ther 2008;120:1-34.

4 Guastella V, Mick G. Strategies for the diagnosis and treatment of neuropathic pain secondary to diabetic peripheral sensory polyneuropathy. Diabetes Metab 2009;35:12-19.

5 Athans W, Stephens H. Open calcaneal fractures in diabetic patients with neuropathy: a report of three cases and literature review. Foot Ankle Int 2008;29:1049-53.

6 Obrosova IG. Diabetic painful and insensate neuropathy: pathogenesis and potential treatments. Neurotherapeutics 2009;6:638-47.

7 Gandhi RA, Marques JLB, Selvarajah D, et al. Painful diabetic neuropathy is associated with greater autonomic dysfunction than painless diabetic neuropathy. Diabetes Care 2010;33:1585-90.

8 Nathan DM, Genuth S, Lachin J, et al. The effect of intensive treatment of diabetes on the development and progression of longterm complications in insulin-dependent diabetes mellitus. N Engl J Med 1993;329:977-86.

9 Duckworth W, Abraira C, Moritz T, et al. Glucose control and vascular complications in veterans with type 2 diabetes. $N$ Engl $J$ Med 2009;360:129-39.

10 Ismail-Beigi F, Craven T, Banerji MA, et al. Effect of intensive treatment of hyperglycaemia on microvascular outcomes in type 2 diabetes: an analysis of the Accord randomised trial. Lancet 2010;376:419-30.

11 Colloca L, Ludman T, Bouhassira D, et al. Neuropathic pain. Nat Rev Dis Primers 2017;3:17002.

12 Javed S, Alam U, Malik RA. Burning through the pain: treatments for diabetic neuropathy. Diabetes Obes Metab 2015;17:1115-25.

13 Elias WJ, Lipsman N, Ondo WG, et al. A randomized trial of focused ultrasound thalamotomy for essential tremor. N Engl J Med 2016;375:730-9.

14 Martínez-Fernández R, Rodríguez-Rojas R, Del Álamo M, et al. Focused ultrasound subthalamotomy in patients with asymmetric Parkinson's disease: a pilot study. Lancet Neurol 2018;17:54-63.

15 Lee Y-F, Lin C-C, Cheng J-S, et al. High-Intensity focused ultrasound attenuates neural responses of sciatic nerves isolated from normal or neuropathic rats. Ultrasound Med Biol 2015;41:132-42.

16 Lee Y-F, Lin C-C, Cheng J-S, et al. Nerve conduction block in diabetic rats using high-intensity focused ultrasound for analgesic applications. Br J Anaesth 2015;114:840-6.

17 Lee Y-F, Lin C-CK, Chen G-S. Temporal course of streptozotocininduced diabetic polyneuropathy in rats. Neurol Sci 2014;35:1813-20

18 Kurokawa K, de Almeida DF, Zhang Y, et al. Sensory nerve conduction of the plantar nerve compared with other nerve conduction tests in rats. Clin Neurophysiol 2004;115:1677-82.

19 Obrosova IG, Xu W, Lyzogubov VV, et al. Parp inhibition or gene deficiency counteracts intraepidermal nerve fiber loss and neuropathic pain in advanced diabetic neuropathy. Free Radic Biol Med 2008;44:972-81.
20 Hussein MR. Evaluation of angiogenesis in normal and lichen planus skin by CD34 protein immunohistochemistry: preliminary findings. Cell Biol Int 2007;31:1292-5.

21 Beliën JA, Somi S, de Jong JS, et al. Fully automated microvessel counting and hot spot selection by image processing of whole tumour sections in invasive breast cancer. J Clin Pathol 1999:52:184-92.

22 Vinik Al, Maser RE, Mitchell BD, et al. Diabetic autonomic neuropathy. Diabetes Care 2003;26:1553-79.

23 Krishnan STM, Quattrini C, Jeziorska M, et al. Abnormal LDIflare but normal quantitative sensory testing and dermal nerve fiber density in patients with painful diabetic neuropathy. Diabetes Care 2009;32:451-5.

24 Schmiedel O, Nurmikko TJ, Schroeter ML, et al. Alpha adrenoceptor agonist-induced microcirculatory oscillations are reduced in diabetic neuropathy. Microvasc Res 2008;76:124-31.

25 Sun P-C, Kuo C-D, Chi L-Y, et al. Microcirculatory vasomotor changes are associated with severity of peripheral neuropathy in patients with type 2 diabetes. Diab Vasc Dis Res 2013;10:270-6.

26 Vinik Al, Holland MT, Le Beau JM, et al. Diabetic neuropathies. Diabetes Care 1992;15:1926-75.

27 Anand P, Terenghi G, Warner G, et al. The role of endogenous nerve growth factor in human diabetic neuropathy. Nat Med 1996;2:703-7.

28 Parkhouse N, Le Quesne PM. Impaired neurogenic vascular response in patients with diabetes and neuropathic foot lesions. $N$ Engl J Med 1988;318:1306-9.

29 Walmsley D, Wiles PG. Early loss of neurogenic inflammation in the human diabetic foot. Clin Sci 1991;80:605-10.

30 Masquelet AC, Penteado CV, Romana MC, et al. The distal anastomoses of the medial plantar artery: surgical aspects (2.10.1987). Surg Radiol Anat 1988;10:247-9.

31 Ozer MA, Govsa F, Bilge O. Anatomic study of the deep plantar arch. Clin Anat 2005;18:434-42.

32 Song CW, Kang MS, Rhee JG, et al. Effect of hyperthermia on vascular function in normal and neoplastic tissues. Ann N Y Acad Sci 1980;335:35-47.

33 Chiesa ST, Trangmar SJ, Kalsi KK, et al. Local temperature-sensitive mechanisms are important mediators of limb tissue hyperemia in the heat-stressed human at rest and during small muscle mass exercise. Am J Physiol Heart Circ Physiol 2015;309:H369-80.

34 Chiesa ST, Trangmar SJ, González-Alonso J. Temperature and blood flow distribution in the human leg during passive heat stress. J Appl Physiol 2016;120:1047-58.

35 Maruo A, Hamner CE, Rodrigues AJ, et al. Nitric oxide and prostacyclin in ultrasonic vasodilatation of the canine internal mammary artery. Ann Thorac Surg 2004;77:126-32.

36 Palmer RM, Ashton DS, Moncada S. Vascular endothelial cells synthesize nitric oxide from L-arginine. Nature 1988;333:664-6.

37 Cameron NE, Eaton SE, Cotter MA, et al. Vascular factors and metabolic interactions in the pathogenesis of diabetic neuropathy. Diabetologia 2001;44:1973-88.

38 Callaghan BC, Cheng HT, Stables CL, et al. Diabetic neuropathy: clinical manifestations and current treatments. Lancet Neurol 2012;11:521-34.

39 Yagihashi S, Mizukami H, Sugimoto K. Mechanism of diabetic neuropathy: where are we now and where to go? J Diabetes Investig 2011;2:18-32.

40 Tuck RR, Schmelzer JD, Low PA. Endoneurial blood flow and oxygen tension in the sciatic nerves of rats with experimental diabetic neuropathy. Brain 1984;107 (Pt 3):935-50.

41 Katz MA, McCuskey P, Beggs JL, et al. Relationships between microvascular function and capillary structure in diabetic and nondiabetic human skin. Diabetes 1989;38:1245-50.

42 Krishnan STM, Quattrini C, Jeziorska M, et al. Neurovascular factors in wound healing in the foot skin of type 2 diabetic subjects. Diabetes Care 2007;30:3058-62.

43 Stehouwer CDA, Dysfunction M. Microvascular dysfunction and hyperglycemia: a vicious cycle with widespread consequences. Diabetes 2018;67:1729-41.

44 Misawa S, Kuwabara S, Kanai K, et al. Axonal potassium conductance and glycemic control in human diabetic nerves. Clin Neurophysiol 2005;116:1181-7.

45 Bestetti G, Rossi GL, Zemp C. Changes in peripheral nerves of rats four months after induction of streptozotocin diabetes. A qualitative and quantitative study. Acta Neuropathol 1981;54:129-34.

46 Lyng H, Monge OR, Bøhler PJ, et al. Relationships between thermal dose and heat-induced tissue and vascular damage after thermoradiotherapy of locally advanced breast carcinoma. Int $J$ Hyperthermia 1991;7:403-15.

47 Dewey WC. Arrhenius relationships from the molecule and cell to the clinic. Int J Hyperthermia 1994;10:457-83. 